\title{
Complexity of Spontaneous QT Variability Unrelated to RR Variations and Respiration During Graded Orthostatic Challenge
}

\author{
Alberto Porta $^{1,2}$, Beatrice Cairo ${ }^{1}$, Beatrice De Maria ${ }^{3}$, Vlasta Bari $^{2}$ \\ ${ }^{1}$ Department of Biomedical Sciences for Health, University of Milan, Milan, Italy \\ ${ }^{2}$ Department of Cardiothoracic, Vascular Anesthesia and Intensive Care, IRCCS Policlinico San \\ Donato, San Donato Milanese, Milan, Italy \\ ${ }^{3}$ IRCCS Istituti Clinici Scientifici Maugeri, Milan, Italy
}

\begin{abstract}
The fluctuations of the duration of the electrical activity of the heart, measured as the time distance between $Q$-wave onset and T-wave end (QT), is under autonomic control. We studied the complexity of the $Q T$ variability regulation via the computation of sample entropy of $Q T$ variability during sympathetic activation induced by graded head-up tilt. Sample entropy was computed over the original QT series and after factorizing it into partial processes describing $Q T$ variability related to heart period, measured as the time interval between consecutive $R$-wave peaks (RR), linked to respiration $(R)$ and unrelated to $R R$ and $R$. We found that $Q T$ variability complexity is high and does not vary with the intensity of the stimulus. This result was the consequence of a non-significant tendency of the complexity of the $Q T$ variability related to $R R$ to decrease and a significant raise of the complexity of the $Q T$ variability unrelated to $R R$ and $R$ with the magnitude of the orthostatic challenge. We suggest that the sample entropy of the $Q T$ variability unrelated to $R R$ and $R$ could quantify the increased heterogeneity of the neural inputs genuinely modulating QT during a sympathetic arousal.
\end{abstract}

\section{Introduction}

Complexity of the beat-to-beat fluctuations of heart period, derived as the time interval between two consecutive R-waves (RR) from the surface electrocardiogram (ECG), decreases during sympathetic activation and vagal withdrawal. For example, a decrease of sample entropy (SampEn) was observed during postural challenge and the reduction was gradual with the magnitude of the stimulus [1]. The time interval between the Q-wave onset and T-wave end (QT) depends largely on the previous RR [2], thus suggesting that QT variability complexity might follow the decrease of the RR variability complexity. At difference with the complexity of RR variability, the complexity of the QT variability in healthy individuals does not decrease during a sympathetic stimulus in healthy subjects [3-5] and it is higher in pathological populations featuring a dominant sympathetic drive compared to normal subjects [6-8]. The issue of estimating the complexity of QT variability is relevant because it was suggested that a limited complexity of the QT variability might lessen the arrhythmic risk $[9,10]$. In the present study we hypothesize that, despite the decrease of the RR variability irregularity during sympathetic activation, QT variability complexity remains high and this behavior is owing to the increase of the complexity of the neural actions genuinely modifying QT [11].

The aim of this study is to assess the complexity of the QT variability after decomposing it into components describing the portion of the QT fluctuations driven by $\mathrm{RR}$, by respiration (R) and unrelated to $\mathrm{RR}$ and $\mathrm{R}$ during a graded orthostatic challenge $[4,12]$. Complexity of QT variability was assessed via SampEn [13].

\section{Methods}

\subsection{QT variability factorization}

First the mean value was subtracted from the series $\mathrm{QT}=\left\{Q T_{n}, n=1, \ldots, N\right\}, \mathrm{RR}=\left\{R R_{n}, n=1, \ldots, N\right\}$, and $\mathrm{R}=\left\{R_{n}\right.$, $n=1, \ldots, N\}$ and each sample was divided by the standard deviation. After normalization the current values $Q T_{n}$ was described as a linear combination of $p$ past values of QT, of the current and $p$ past values of $\mathrm{RR}$ and $\mathrm{R}$ plus a sample of an autoregressive noise $\eta_{n}[4]$ as

$$
Q T_{n}=A_{Q T}(z) \cdot Q T_{n}+B(z) \cdot R R_{n}+C(z) \cdot R_{n}+\eta_{n},
$$

where $\quad A_{Q T}(z) \cdot=\sum_{i=1}^{p} a_{Q T, i} \cdot z^{-i}, \quad B(z) \cdot=\sum_{i=0}^{p} b_{i} \cdot z^{-i}, \quad$ and $C(z) \cdot=\sum_{i=0}^{p} c_{i} \cdot z^{-i}$ are polynomials in the $Z$-domain with $z^{-1}$ 
being the one-step delay operator and $\eta_{n}=D(z) \cdot \eta_{n}+w_{Q T, n}$ where $w_{Q T, n}$ is a sample of white Gaussian noise and $D(z) \cdot=\sum_{i=1}^{p} d_{i} \cdot z^{-i}$. According to an open loop approach [14] the current values of $R R_{n}$ and $R_{n}$ are modelled as realization of an univariate autoregressive process, namely $R R_{n}=A_{R R}(z) \cdot R R_{n}+w_{R R, n} \quad$ and $R_{n}=A_{R}(z) \cdot R_{n}+w_{R, n}, \quad$ where $\quad A_{R R}(z) \cdot=\sum_{i=1}^{p} a_{R R, i} \cdot z^{-i}$ and $A_{R}(z) \cdot=\sum_{i=1}^{p} a_{R, i} \cdot z^{-i}$ are polynomials in the $Z$-domain and $w_{R R, n}$ and $w_{R, n}$ are samples of white Gaussian noises. The coefficients $a_{Q T, i}, b_{i}, c_{i}$, and $d_{i}$ were estimated via generalized least squares approach with stopping criterion over the variation of prediction error (threshold $=0.001$ ), while $a_{R R, i}$ and $a_{R, i}$ via traditional least squares approach. Both generalized and traditional least squares problems were solved via Cholesky decomposition method $[4,15]$. The model order $p$ was optimized via the Akaike figure of merit for multivariate processes in the range from 4 and 16 [4,15]. The QT series was factorized into three partial processes $\mathrm{QT}_{\mathrm{wRR}}, \mathrm{QT}_{\mathrm{wR}}$ and $\mathrm{QT}_{\mathrm{wQT}}$ representing the portions of QT variability depending on $\mathrm{RR}, \mathrm{R}$ and unrelated to $\mathrm{RR}$ and $\mathrm{R}$ respectively [4] with

$$
Q T_{n}=Q T_{w_{R R}, n}+Q T_{w_{R}, n}+Q T_{w_{Q T}, n} .
$$

$\mathrm{QT}_{\mathrm{wRR}}, \mathrm{QT}_{\mathrm{wR}}$ and $\mathrm{QT}_{\mathrm{wQT}}$ were obtained by filtering, respectively, $\mathrm{w}_{\mathrm{RR}}, \mathrm{w}_{\mathrm{R}}$ and $\mathrm{w}_{\mathrm{QT}}$ with

$$
\begin{aligned}
& H_{Q T-R R}(z)=B(z) \cdot\left[1-A_{Q T}(z)\right]^{-1} \cdot\left[1-A_{R R}(z)\right]^{-1}, \\
& H_{Q T-R}(z)=C(z) \cdot\left[1-A_{Q T}(z)\right]^{-1} \cdot\left[1-A_{R}(z)\right]^{-1}, \\
& H_{Q T-Q T}(z)=\left[1-A_{Q T}(z)\right]^{-1} \cdot[1-D(z)]^{-1} .
\end{aligned}
$$

The factorization held under the hypotheses of whiteness and uncorrelation of $w_{R R}, w_{R}$, and $w_{Q T}[4,15]$.

\subsection{SampEn}

SampEn assesses the complexity of $\mathrm{x}$ with $\mathrm{x}=\mathrm{RR}$, QT, $\mathrm{QT}_{\mathrm{wRR}}, \mathrm{QT}_{\mathrm{wR}}$, or $\mathrm{QT}_{\mathrm{wQT}}$ via the computation of the amount of information associated to $x_{n}$ that cannot be derived from $d$ past values $x_{n}^{-}=\left(x_{n-1}, \ldots, x_{x-d}\right)$. SampEn is computed [13] as

$$
\operatorname{SampEn}(d, r, N)=-\log \frac{\left\langle p\left(x_{n} \oplus x_{n}^{-}\right)>\right.}{<p\left(x_{n}^{-}\right)>}
$$

where $p\left(x_{n} \oplus x_{n}^{-}\right)$and $p\left(x_{n}^{-}\right)$represent the probability of finding a pattern in the neighborhood of $x_{n} \oplus x_{n}^{-}=\left(x_{n}, x_{n-1}, \ldots, x_{n-d}\right)$ and $x_{n}^{-}$of size $r$ computed by dividing the number of matches by the total number of patterns and the operator $\langle\cdot\rangle$ performs the mean over the time index $n$. The tolerance $r$ was set to $0.2 \times$ standard deviation of $\mathrm{x}$ and $d=3$.

\section{Protocol and preprocessing}

\subsection{Experimental protocol}

The database was exploited to evaluate the performance of open loop versus closed loop modelling structures in describing cardiovascular control [14]. Therefore, we refer to [14] for the full description of the protocol. The study adheres to the principles of the Declaration of Helsinki for medical research involving human subjects. The human research and ethical review boards of the 'Luigi Sacco' Hospital, Milan, Italy approved the protocol. Written informed consent was
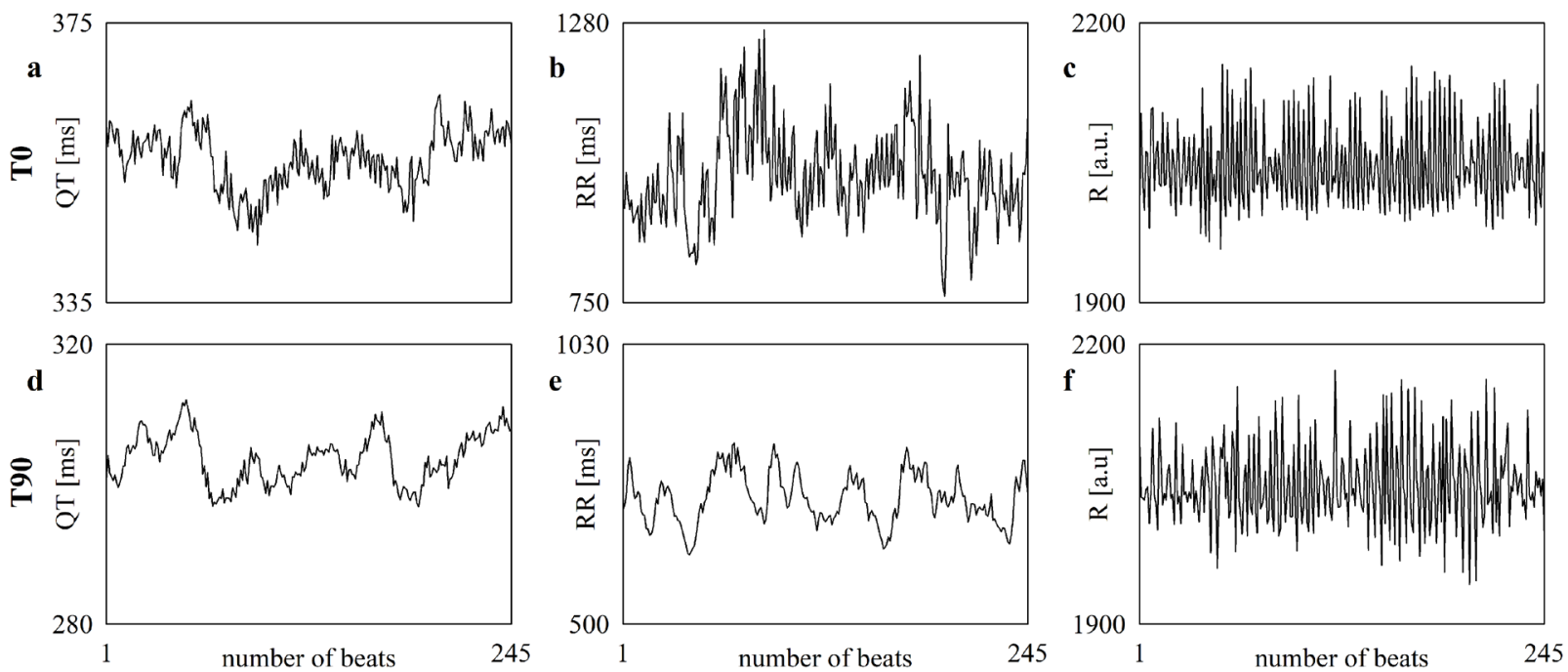

Figure 1. The line plots show examples of QT $(\mathrm{a}, \mathrm{d}), \mathrm{RR}(\mathrm{b}, \mathrm{e})$, and $\mathrm{R}(\mathrm{c}, \mathrm{f})$ series recorded from the same subject during T0 $(\mathrm{a}, \mathrm{b}, \mathrm{c})$ and during T90 (d,e,f). 

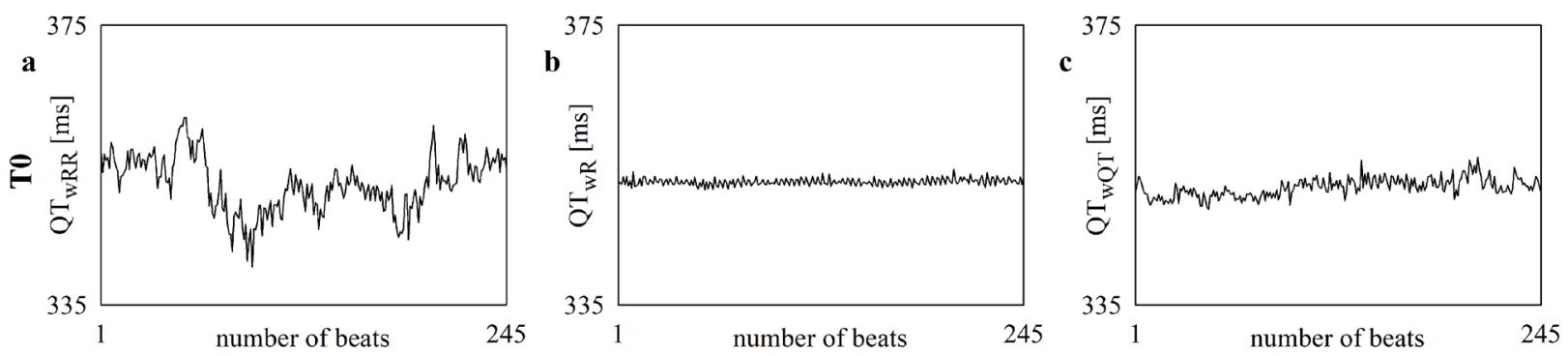

Figure 2. The line plots show the decomposition of the QT series given in Fig.1a into $\mathrm{QT}_{\mathrm{wRR}}(\mathrm{a}), \mathrm{QT}_{\mathrm{wR}}(\mathrm{b})$ and $\mathrm{QT}_{\mathrm{wQT}}(\mathrm{c})$. obtained from all the subjects. Briefly, 15 healthy nonsmoking humans (aged from 24 to 54 years, median = 28 years; 9 males) participated in the study. We acquired ECG (Biosignal Conditioning Device, Marazza, Monza, Italy) from lead II and respiratory movements via a piezoelectric thoracic belt (Marazza, Monza, Italy). Signals were sampled at $1000 \mathrm{~Hz}$. We recorded signals for 7 min at rest in supine position with a tilt table angle of $0^{\circ}$ (T0) and for 10 min during head-up tilt with tilt table angles randomly chosen within the set $\left\{15^{\circ}, 30^{\circ}\right.$, $\left.45^{\circ}, 60^{\circ}, 75^{\circ}, 90^{\circ}\right\}$ (T15, T30, T45, T60, T75, T90). Each head-up tilt session was preceded by a $\mathrm{T} 0$ phase. The full sequence of angles was completed by all subjects without experiencing any sign of presyncope. During the protocol the subjects breathed spontaneously but they were not allowed to talk.

\subsection{Beat-to-beat series extraction}

R-wave peaks were located according to a threshold on the first derivative and parabolic interpolation. The time distance between consecutive R-wave peaks was taken as RR. The T-wave end was located over the T-wave downslope where the absolute first derivative went below a threshold set as a $30 \%$ of the absolute maximal first derivative computed over the current $\mathrm{T}$-wave downslope [16]. QT was approximated as the time distance between the R-wave peak and T-wave end [16]. The $n$th QT intervals followed the $n$th RR. The R signal was sampled in correspondence of the first R-wave peak delimiting the $n$th HP. If an automatic detection of R-wave or T-wave apex was judged to be erroneous or missing, the automatic procedure was run again locally. If isolated ectopic beats were detected, the abnormal values were substituted with their linear interpolation using the closest
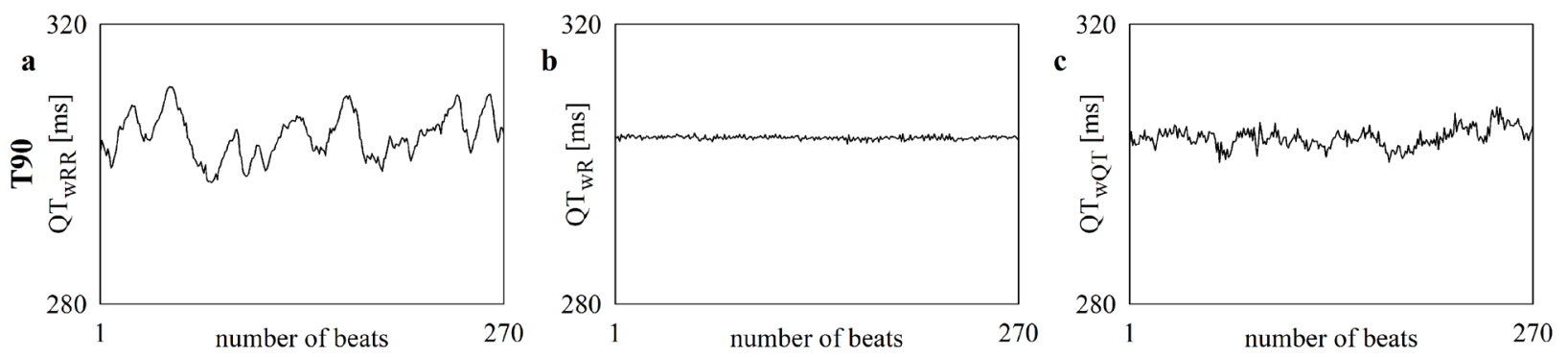

Figure 3. The line plots show the decomposition of the QT series given in Fig.1d into $\mathrm{QT}_{\mathrm{wRR}}(\mathrm{a}), \mathrm{QT}_{\mathrm{wR}}(\mathrm{b})$ and $\mathrm{QT} \mathrm{T}_{\mathrm{wQT}}(\mathrm{c})$. 


\section{Discussion}

The decrease of complexity of RR variability during a sympathetic activation is driven by the reduction of respiratory sinus arrhythmia and the concomitant rise of low frequency oscillations [1]. The complexity of QT variability regulation is the result not only of the neural inputs directed to the sinus node affecting QT dynamics via the QT-RR relation [2] but also of neural actions genuinely acting on the ventricles $[4,12]$ and non-neural influences such as cardiac axis respiratory-related oscillations inducing synchronous changes on the projection of the electrocardiographic activity onto the assigned lead $[4,16]$. When the complexity of the QT regulation was assessed from the QT series, it was found to be greater than that of RR $[5,9,10]$. However, given that QT dynamics depend on RR changes [2], it might be hypothesized that, in presence of a sympathetic activation reducing the RR variability complexity, the QT variability complexity diminishes as well. Conversely, this study demonstrates that the increase of the complexity of QT variability unrelated to R and RR offsets the non-significant tendency of the RR-related QT variability complexity to decrease. Given that the QT variability unrelated to $\mathrm{R}$ and $\mathrm{RR}$ could be mainly attributed to the action of neural inputs directed to ventricles, we suggest that the heterogeneity of these inputs increases during a sympathetic activation. This rise contributes in keeping the complexity of QT variability high $[5,9,10]$ and in decreasing the RR-QT coupling strength during sympathetic activation $[17,18]$.

\section{Conclusions}

Sympathetic activation increases the heterogeneity of neural inputs genuinely modulating QT. The systematic assessment of the complexity of the QT variability unrelated to $R R$ and $R$ might provide new clues for the estimation of the arrhythmic risk linked to sympathetic arousals

\section{References}

[1] A. Porta et al., "Progressive decrease of heart period variability entropy-based complexity during graded headup tilt," J. Appl. Physiol., vol. 103, pp. 1143-1149, 2007.

[2] H. C. Bazett "An analysis of the time-relations of electrocardiograms," Heart, vol. 7, pp. 353-370, 1920.

[3] M. J. Lewis and A. L. Short, "Sample entropy of electrocardiographic RR and QT time-series data during rest and exercise," Physiol. Meas., vol. 28, pp. 731-744, 2007.

[4] A. Porta et al., "RT variability unrelated to heart period and respiration progressively increases during graded head-up tilt," Am. J. Physiol., vol. 298, pp. H1406-H1414, 2010.

[5] M. Baumert et al., "Entropy analysis of RR and QT interval variability during orthostatic and mental stress in healthy subjects," Entropy, vol. 16, pp. 6384-6393, 2014.

[6] Sosnowski et al., "Scatterplots of RR and RT interval variability bring evidence for diverse non-linear dynamics of heart rate and ventricular repolarization duration in coronary heart disease," Europace, vol. 3, pp. 39-45, 2001.

[7] E. Nahshoni et al., "Complexity of the dynamic QT variability and RR variability in patients with acute anterior wall myocardial infarction: a novel technique using a nonlinear method," J. Electrocardiol., vol. 37, pp. 173-179, 2004.

[8] Y. Li et al., Variability in patients with coronary artery disease and congestive heart failure: a comparison with healthy control subjects," Med. Biol. Eng. Comput., vol. 57, pp. 389-400, 2019.

[9] V. Bari et al., "Low-pass filtering approach via empirical mode decomposition improves short-scale entropy-based complexity estimation of QT interval variability in long QT syndrome type 1 patients," Entropy, vol. 16, pp. 4839 4854, 2014.

[10] V. Bari et al., "Multiscale complexity analysis of the cardiac control identifies asymptomatic and symptomatic patients in long QT syndrome type 1," PLOS ONE, vol. 9, Art. no. e93808, 2014.

[11] M. T. La Rovere et al., "Autonomic control of the heart and its clinical impact. A personal perspective," Front. Physiol., vol. 11, Art. no. 582, 2020

[12] A. Porta et al., "Quantifying electrocardiogram RT-RR variability interactions," Med. Biol. Eng. Comput., vol. 36, pp. 27-34, 1998

[13] J. S. Richman, and J. R. Moorman, "Physiological timeseries analysis using approximate entropy and sample entropy," Am. J. Physiol., vol. 278, pp. H2039-H2049, 2000.

[14] A. Porta et al., "Model-based assessment of baroreflex and cardiopulmonary couplings during graded head-up tilt," Comput. Biol. Med., vol. 42, pp. 298-305, 2012.

[15] G. Baselli et al., "Spectral decomposition in multichannel recordings based on multi-variate parametric identification," IEEE Trans. Biomed. Eng., vol. 44, pp. 1092-1101, 1997.

[16] A. Porta et al., "Performance assessment of standard algorithms for dynamic R-T interval measurement: comparison between R-Tapex and R-Tend approach," Med. Biol. Eng. Comput., vol. 36, pp. 35-42, 1998.

[17] A. Porta et al., "Frequency domain assessment of the coupling strength between ventricular repolarization duration and heart period during graded head-up tilt," $J$. Electrocardiol., pp. 44, vol. 662-668, 2011.

[18] A. Porta et al., "A network physiology approach to the assessment of the link between sinoatrial and ventricular cardiac controls," Physiol. Meas., vol. 38, pp. 1472-1489, 2017.

Address for correspondence:

Prof. Alberto Porta, $\mathrm{PhD}$

Università degli Studi di Milano

Dipartimento di Scienze Biomediche per la Salute

IRCCS Policlinico San Donato

Via F. Fellini 4, 20097, San Donato Milanese, Milan, Italy

Tel: +39 02 52774382; email: alberto.porta@unimi.it 